\title{
1 Is the perception of illusions abnormal in schizophrenia?
}

3 Lukasz Grzeczkowskia,b, Maya Roinishvili ${ }^{,}$, , Eka Chkonia ${ }^{\circ}$, Andreas Brand, Fred W.Mast ${ }^{g}$,

4 Michael H. Herzog ${ }^{a}$, Albulena Shaqiri ${ }^{a}$

5 'Laboratory of Psychophysics, Brain Mind Institute, École Polytechnique Fédérale de Lausanne (EPFL),

6 Switzerland

$7 \quad{ }^{b}$ Allgemeine und Experimentelle Psychologie, Department Psychologie, Ludwig-Maximilians-Universität

8 München (LMU), Germany

$9{ }^{c}$ Laboratory of Vision Physiology, Beritashvili Centre of Experimental Biomedicine, Tbilisi, Georgia

$10{ }^{d}$ Institute of Cognitive Neurosciences, Free University of Tbilisi, Tbilisi, Georgia

11 'Department of Psychiatry, Tbilisi State Medical University, Tbilisi, Georgia

12 Institute of Psychology and Cognition Research, University of Bremen, Germany

$13{ }^{g}$ Department of Psychology, University of Bern, Switzerland

15 Corresponding Author:

16 Lukasz Grzeczkowski

17 Ludwig-Maximilians-Universität

18 Department Psychologie

19 Leopoldstraße 13

2080802 München

21 Germany

22 lukasz.grzeczkowski@gmail.com 
There seems to be no common factor for visual perception, i.e., performance in visual tasks correlates only weakly with each other. Similar results were found with visual illusions. One may expect common visual factors for individuals suffering from pathologies that alter brain functioning, such as schizophrenia. For example, patients who are more severely affected by the disease, e.g., stronger positive symptoms, may show increased illusion magnitudes. Here, in the first experiment, we used a battery of seven visual illusions and a mental imagery questionnaire. Illusion magnitudes for the seven illusions did not differ significantly between the patients and controls. In addition, correlations between the different illusions and mental imagery were low. In the second experiment, we tested 59 patients (mostly outpatients) with ten visual illusions. As for the first experiment, patients and controls showed similar susceptibility to all but one visual illusion. Moreover, there were no significant correlations between different illusions, symptoms, or medication type. Thus, it seems that perception of visual illusions is mostly intact in schizophrenia.

\section{Introduction}

Numerous studies have tested illusion strength in schizophrenia patients. Results have been mixed and depend on the illusion tested (for a review, see King et al., 2016). For example, studies have found that patients perceive some illusions, such as the Müller-Lyer or the Ponzo illusion, significantly more strongly than controls (Weckowicz and Witney, 1960; Capozzoli and Marsh, 1994; Chen et al., 2011; Diržius et al., 2013; Kantrowitz et al., 2009; Tam et al., 1998). Other studies found non-significant results for the Ebbinghaus illusion and the illusory Kanizsa squares (Kantrowitz et al., 2009; Spencer and Ghorashi, 2014; Tibber et al., 2013; Yang et al., 2013), and some studies have reported that illusion magnitudes of the contrast-contrast illusion, the illusory line motion, and the Hollow mask illusion are significantly weaker in the patients (Barch et al., 
al., 2013; Letourneau, 1974; Parnas et al., 2001; Robol et al., 2013; Sanders et al., 2013; Schneider et al., 2002; Tadin et al., 2006; Tam et al., 1998; Tibber et al., 2013; Uhlhaas et al., 2006; Yang et al., 2013). Even when it comes to the same illusions, results sometimes differ. For example, some studies found that patients are less influenced by the Ebbinghaus illusion than controls (Tibber et al., 2013; Uhlhaas et al., 2006), whereas Yang et al., (2013) found nonsignificant results. Similarly, for the Müller-Lyer illusion, both significant and non-significant differences were reported (for a review, see King et al., 2016). Sample sizes are rather small in many of these studies, having on average between 15 and 30 patients, so it may be that the mixed results are a matter of heterogeneous samples and a lack of power. For some of these nonsignificant results, it is hence unclear whether they reflect "true" null results or whether the studies are underpowered (e.g., Letourneau, 1974, $\mathrm{n}=5$; Parnas et al., 2001, $\mathrm{n}=10$; Spencer and Ghorashi, 2014, $\mathrm{n}=17$; Tibber et al., 2013, $\mathrm{n}=24$ ).

Recently in a sample of 144 healthy controls, we found that illusion magnitudes correlated only weakly with each other (Grzeczkowski et al., 2017). It seems that there is no common factor for visual illusions. As a side note, there were also only weak correlations in standard visual tests in young, healthy adults (Bosten and Mollon, 2010; Cappe et al., 2014) and in healthy aging (Shaqiri et al., 2015). Here, we investigated whether there is a common cause for perception of visual illusions in schizophrenia. Such a common factor could be related to the strength of visual hallucinations (Bracha et al., 1989; Ford et al., 2015; Goghari and Harrow, 2016) and increased mental imagery (Brébion et al., 2000, 1997; Oertel et al., 2009; Sack et al., 2005). Waters et al., (2014) report that more than $27 \%$ of schizophrenia patients suffer from visual hallucinations. Moreover, schizophrenia patients were found to have a reality-monitoring deficit (Brune, 2005; Frith and Corcoran, 1996), i.e., a decreased ability to discriminate real events from imagined events (Brébion et al., 2000, 1997; Oertel et al., 2009; Sack et al., 2005).

To this end, we used a battery of seven illusions and a questionnaire about the vividness of mental imagery in a first experiment with a sample of 19 schizophrenia patients and 19 controls. 
In a second experiment, with a sample of 59 schizophrenia patients and 54 controls, we tested the susceptibility to ten visual illusions. Often, illusions strength is determined with binary judgments where a reference element, such as the central disk in the Ebbinghaus illusion, and a slightly larger or smaller disk are presented (King et al., 2016). Participants indicate whether this disk is larger or smaller than the reference disk. In these experiments, stimuli are hard to discriminate to obtain good estimates, and thus, attention is a crucial component. However, attention is often deficient in the patients, adding a confounding factor (Chkonia et al., 2010; Perlstein et al., 1998). To avoid this problem, we used an adjustment procedure, where participants adjusted their precepts with the computer mouse.

To preface our results, we found almost no significant differences between controls and patients in illusion magnitudes nor in the vividness of mental imagery. In addition, illusion magnitudes of the patients correlated only weakly with each other, and this was also true for controls. We suggest that the perception of illusions is largely intact in patients (for a review see King et al., 2016; Notredame et al., 2014).

\section{General Methods}

\subsection{Participants}

Participants were schizophrenia patients either from the Tbilisi Mental Health Hospital or the psychosocial rehabilitation center and healthy, age and education-matched controls, from the general population from Tbilisi (see Table 1 for details). Patients were diagnosed according to DSM-IV by means of an interview based on the SCID, information of the staff, and the study of the records. Psychopathology of schizophrenia patients was assessed by an experienced psychiatrist (EC) by Scales for the Assessment of Negative Symptoms and Scales for the Assessment of Positive Symptoms (SANS, SAPS; Andreasen, 1984a, 1984b). All participants had visual acuity of equal or greater than 0.8 for at least one eye, as measured with the Fribourg Visual Acuity Test (Bach, 1996). All participants signed informed consent before the experiment. 
100 All procedures were in accordance with the Declaration of Helsinki and were approved by The

101 Georgian National Council on Bioethics in Tbilisi.

102

103

104

105

106

107

108

109

110

111

112

113 the Psychophysics toolbox (Brainard, 1997; Pelli, 1997). Participants sat at $\sim 60 \mathrm{~cm}$ from the 114 screen and used a Logitech LS1 computer mouse for stimuli adjustments.

\begin{tabular}{ccccc|cccc} 
& \multicolumn{5}{c|}{ Exp. 1 } & \multicolumn{5}{c}{ Exp. 2 } \\
\cline { 2 - 9 } & SZ & HC & $t_{\text {[d] }]}$ & $p$ & SZ & HC & $t_{[d]}$ & $p$ \\
\hline Sex (F/M) & $5 / 14$ & $5 / 14$ & - & - & $14 / 45$ & $26 / 28$ & - & - \\
Age & $49 \pm 9.3$ & $40 \pm 6.6$ & $0.42_{[36]}$ & 0.675 & $38 \pm 8.3$ & $38 \pm 9.2$ & $0.15_{[108]}$ & 0.881 \\
Age range & $22-53$ & $27-50$ & - & - & $22-55$ & $24-55$ & - & - \\
Education & $13.3 \pm 2.5$ & $15 \pm 2.8$ & $2.08_{[36]}$ & 0.045 & $13.3 \pm 2.4$ & $15.3 \pm 2.5$ & $4.14_{[100]}$ & $<0.001$ \\
VVIQ & $104 \pm 22$ & $118 \pm 25$ & $1.94[36]$ & 0.060 & - & - & - & - \\
SAPS & $9.3 \pm 3.5$ & - & - & - & $8.4 \pm 2.3$ & - & - & - \\
SAPS range & $3-16$ & - & - & - & $4-17$ & - & - & - \\
SANS & $10 \pm 5.3$ & - & - & - & $10 \pm 5.1$ & - & - & - \\
SANS range & $0-20$ & - & - & - & $2-20$ & - & - & - \\
Illness duration & $14 \pm 9$ & - & - & - & $14.5 \pm 8$ & - & - & - \\
CPZ & $610 \pm 387$ & - & - & - & $641 \pm 418$ & - & - & - \\
Patients (in/out) & $8 / 11$ & - & - & - & $11 / 53$ & - & - & - \\
\hline
\end{tabular}

\subsection{Apparatus}

Experiment 1 was performed on a Dell Latitude E5540 computer with a 15-inch screen. Experiment 2 was performed on a desktop computer, equipped with a 24-inch, BenQ XL2420T monitor. In both experiments, the screen resolution was set up to 1920 x 1080 pixels and was refreshed at a rate of $60 \mathrm{~Hz}$. Stimuli were generated with Matlab 2013b (version 3.1, 64 bits) and 
116 First, all illusions were shown one by one on the screen and the adjustment procedure was

117 explained by the experimenter. For each illusion, participants compared a reference stimulus to a 118 target stimulus that they adjusted by displacing the computer mouse on its horizontal axis. Each 119 participant performed two trials per illusion without time restrictions. Illusions were adjusted in 120 the same order by each participant: Ebbinghaus, Müller-Lyer, Ponzo, simultaneous contrast, 121 Ponzo "hallway", White's and tilt for Exp. 1; and Ebbinghaus 1, Ebbinghaus 2 "small", 122 Ebbinghaus 3 "big", Müller-Lyer, Ponzo, Ponzo "wide", simultaneous contrast, Ponzo "grid", 123 White's and tilt for Exp. 2. Participants were asked to make their adjustments relying on their 124 perception and to ignore any prior knowledge they may have had of visual illusions. At the end of each experiment, participants were debriefed and they could see their own results on the computer screen.

\subsection{Data Analysis}

128 For each participant and trial, the raw data was transformed into percentage of error in the following manner: for all illusions, except the tilt illusion, the reference value of the disk diameter, length, or luminance was subtracted from the adjusted stimulus diameter, length, or luminance. That difference was then divided by the value of the reference stimulus and multiplied by 100. Similarly, for the tilt illusion, the reference angle (33 degrees) was first subtracted from the adjusted angle, then divided by the maximum possible bias (i.e., range between the inner and

134 the surround orientation of the inducer stimulus $=69$ degrees). Thus, for the tilt illusion, 1 degree 135 of error corresponds to $1.45 \%$ of error. Therefore, an adjusted size, length, luminance, or angle 136 that perfectly corresponded to the reference stimulus has a value of zero percentage of bias. To 137 the contrary, $100 \%$ of bias would correspond to a doubled reference value (e.g., reference 138 stimulus length $=4 \mathrm{deg}$, adjusted stimulus length $=8 \mathrm{deg}$ ). 


\section{Experiment 1}

\subsection{Stimuli}

The illusion magnitudes for seven visual illusions were determined: Ebbinghaus illusion (EB), Müller-Lyer illusion (ML), Ponzo illusion (PZ), simultaneous contrast illusion (SC), Ponzo "hallway" illusion (PZh), White's illusion (WH), and tilt (TT) illusion (Figure 1). The reference stimulus for the Ebbinghaus, Müller-Lyer and tilt illusions was centered at 8 degrees to the left from the center of the screen and the adjustable stimulus at 8 degrees to the right.

\subsubsection{Ebbinghaus Illusion (EB)}

The reference was a white disk of 2 degrees in diameter, surrounded by sixteen smaller yellow disks (inducers), 0.5 degrees of diameter each. The distance between the centers of the reference disk and the small inducers was 1.6 degrees. Large inducers, surrounding the adjustable disk were 4 degrees in diameter. The distance between the center of the adjustable disk and the center of each large inducer was 5 degrees. At the beginning of each trial, the adjustable disk appeared with a random size in the range of 0 to 6 degrees in diameter. Both the luminance of the yellow surrounding disks and the white central disks was $128 \mathrm{~cd} / \mathrm{m}^{2}$. The background luminance was 1 $\mathrm{cd} / \mathrm{m}^{2}$.

\subsubsection{Müller-Lyer illusion (ML)}

The length of the reference line was 5.4 degrees and it was always presented with inward-pointing arrows. The lines composing the arrows were 1-degree long. The adjustable line was always presented with outward-pointing arrows and its starting length varied randomly between 0 and 16 degrees. The line's luminance was $128 \mathrm{~cd} / \mathrm{m}^{2}$.

\subsubsection{Ponzo illusion (PZ)}

The reference stimulus was the yellow $\left(128 \mathrm{~cd} / \mathrm{m}^{2}\right), 3$ degrees long, horizontal, lower line. The adjustable line was the horizontal, upper yellow line. The initial length of the adjustable line varied randomly from trial to trial but never extended beyond 16 degrees. Both the reference and 
164 the adjustable lines were centered on the vertical midline of the screen and were placed at 3 165 degrees from the horizontal screen midline. The ends of the white diagonal lines (inducers) were 166 placed at 3.8 degrees from the horizontal screen midline. The distances between the two upper 167 and lower line ends were 3 and 7.6 degrees respectively.

\subsubsection{Simultaneous contrast illusion (SC)}

169 The reference and the adjustable stimuli were small squares with a side-length of 2.6 degrees 170 placed at 3.9 degrees to the left and right of the screen center, respectively. The luminance of the reference square was $35 \mathrm{~cd} / \mathrm{m}^{2}$. These small squares were embedded in bigger, 7.8 degree squares. The luminance of the big square placed on the left was $15 \mathrm{~cd} / \mathrm{m}^{2}$ and $70 \mathrm{~cd} / \mathrm{m}^{2}$ for the one on the right.

\subsubsection{Ponzo "hallway" illusion (PZh)}

175

The diameter of the reference disk was 1.6 degrees. The disk was located on the top-right hand corner, 14.4 degrees from the screen's center. The adjustable disk appeared on the lower-left hand corner, 10.8 degrees from the screen's center. The luminance of both disks was $15 \mathrm{~cd} / \mathrm{m}^{2}$. During the adjustment, the lowest point of the adjustable disk was fixed while its center moved up. This created the impression that the disk was anchored to the image background. The background image was a 1920 x 1080 pixel resolution grayscale picture of a hallway at the EPFL campus.

\subsubsection{White's illusion (WH)}

182 The background was composed of alternating dark $\left(1 \mathrm{~cd} / \mathrm{m}^{2}\right)$ and light $\left(128 \mathrm{~cd} / \mathrm{m}^{2}\right)$ horizontal, 1831.75 degree wide stripes. The gray reference rectangles on the left were 1.75 degrees tall and 3.6 184 degrees wide. They were presented on light bands and their luminance was $15 \mathrm{~cd} / \mathrm{m}^{2}$. The adjustable rectangles appearing on the right lay on dark bands and were the same size as their reference counterparts. All rectangles were at 1.6 degrees from the screen's vertical meridian. During adjustments, the rightward rectangles changed gradually in luminance, with a starting luminance chosen randomly at the beginning of each trial from between 0 and $128 \mathrm{~cd} / \mathrm{m}^{2}$. 
190 The reference and the adjustable stimuli were disks with a diameter of 4 degrees, each containing 191 a 0.5 cycles/deg full contrast grating texture. The reference disk was tilted 33 degrees towards the 192 clockwise direction from vertical and was embedded in a larger disk (13 degrees in diameter) with 193 the same spatial frequency but tilted 36 degrees towards the counter-clockwise direction. The 194 background luminance was $15 \mathrm{~cd} / \mathrm{m}^{2}$. The adjustable disk appeared with a random orientation 195 between 0 and 360 degrees.
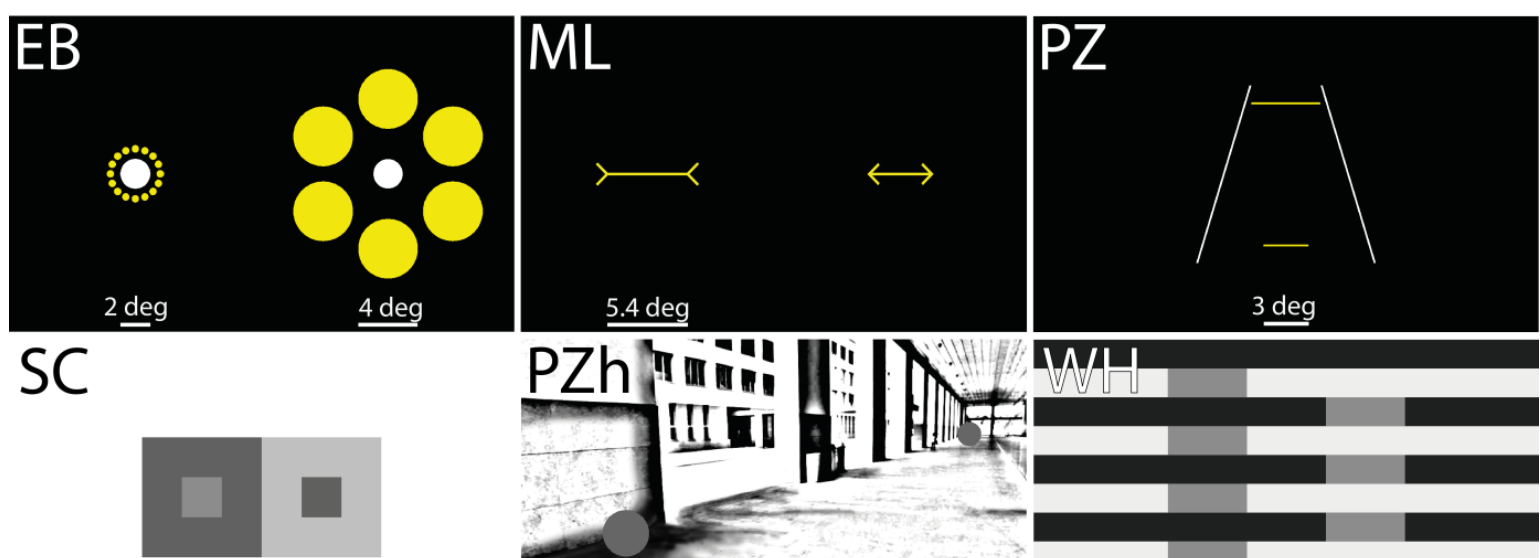

2.6 deg $5.2 \mathrm{deg}$
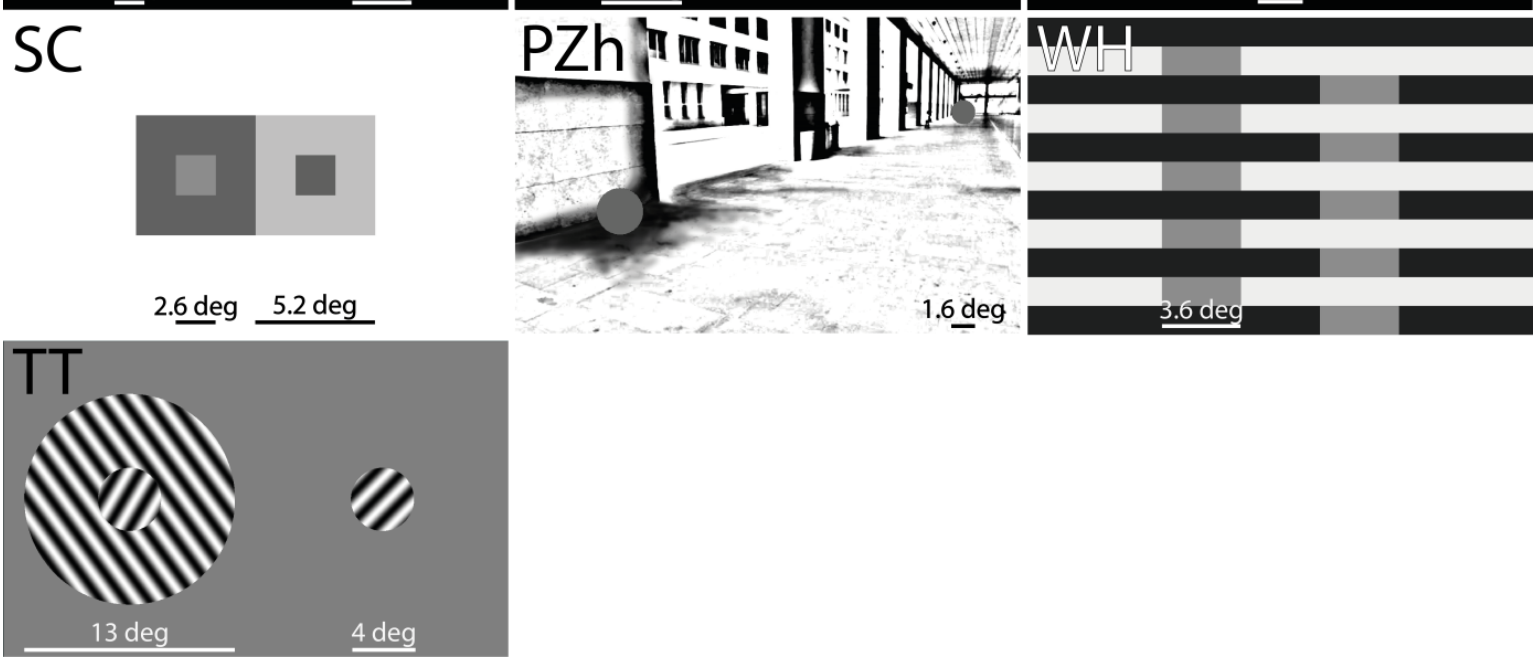

197 Figure 1. The seven visual illusions used in Exp. 1. In the Ebbinghaus illusion (EB), participants adjusted 198 the size of the right white disk to the size of the white disk on the left. In the Müller-Lyer illusion (ML), participants adjusted the length of the line on the right to the one on the left. In the Ponzo (PZ) illusion, participants adjusted the length of the upper horizontal yellow line to match that of the lower horizontal yellow line. In the simultaneous contrast illusion (SC), participants adjusted the luminance of the right center square to the left center square. In the Ponzo "hallway" illusion (PZh), participants adjusted the size of the lower-left gray disk to that of the upper-right gray disk. In the White's illusion (WH), participants adjusted the luminance of gray bars on the right to the luminance of the bars on the left. In the tilt illusion 
205 (TT), participants adjusted the orientation of the right disk to that of the left disk embedded in the counter-

206 clockwise tilted surround. For each illusion, participants performed two adjustment trials.

\subsection{Vividness of visual imagery questionnaire}

208 Prior to the illusion magnitude assessments, participants completed the vividness of visual imagery questionnaire (VVIQ; Marks, 1973). Participants were asked to generate mental images described in each of sixteen items, and then to estimate the vividness of these mental images by

211 circling the corresponding number in a five-point scale (1 - no image at all, you only « know » you 212 are thinking of an object; 2 - vague and dim; 3 - moderately clear and vivid; 4 - clear and 213 reasonably vivid; 5 - perfectly clear and vivid as normal vision). The VVIQ was first completed 214 with open- and then with closed-eyes when generating mental images. Scores from both eyes 215 were summed to give a final VVIQ score.

\subsection{Results}

\section{$217 \quad 2.3 .1 \quad$ Test-retest reliability}

218 We determined illusions magnitude with 2 trials for each observer. To determine test-retest 219 reliability, we correlated the two trials. Test-retest reliability was highly significant for the control 220 group for all the seven illusions (Table 2, second row). For the schizophrenia patients (Table 2, 221 first row), correlations were significant for five out of seven illusions, but not for the Müller-Lyer 222 and the White's illusion. After correction for multiple comparisons (Bonferroni, $p=0.0036$ ), all 223 significant correlations except for the tilt illusion remained significant. Test-retest reliability of 224 VVIQ is high (e.g., Burton and Fogarty, 2003). 
225 Table 2. Test-retest reliability expressed as Bravais-Pearson's $R$ correlations between the first and the 226 second trial for seven visual illusions for schizophrenia patients (SZ, first row) and age-matched, healthy 227 controls (HS, second row).

\begin{tabular}{|c|c|c|c|c|c|c|c|}
\hline & EB & $\mathrm{ML}$ & $\mathrm{PZ}$ & $\mathrm{SC}$ & $\mathrm{PZh}$ & WH & TT \\
\hline $\mathrm{SZ}$ & $.78 \star \star \star$ & .19 & $.85 \star \star \star$ & $.87 \star \star \star$ & $.89 \star \star \star$ & .44 & $.58 \star \star$ \\
\hline $\mathrm{HC}$ & $.82 \star \star \star$ & $.84 \star \star \star$ & $.90 \star \star \star$ & $.89 \star \star \star$ & $.98 \star \star \star$ & $.76 \star \star \star$ & $.74 \star \star \star$ \\
\hline
\end{tabular}

\subsubsection{Illusion magnitudes}

229 Next, we averaged the illusion magnitudes for the 2 trials (Figure 2). Independent samples $t$-tests 230 were performed to compare illusion magnitudes between controls and patients separately for each 231 illusion. None of the comparisons was significantly different. Corrections for multiple 232 comparisons were not applied.
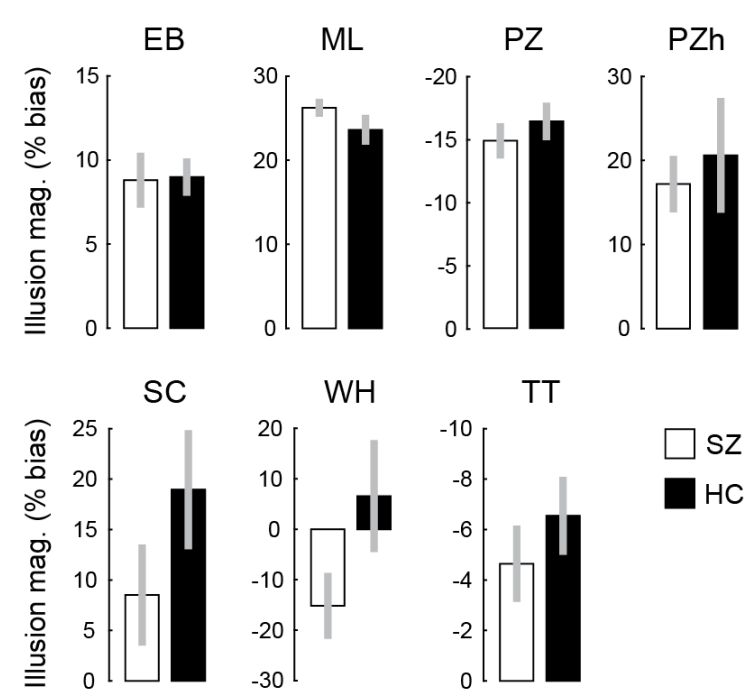

234 Figure 2. Average illusion magnitudes for healthy controls (HC, white) and schizophrenia patients (SZ, black). Illusion magnitudes were not significantly different between patients and 236 controls. We did not apply Bonferroni corrections for multiple comparisons. Hence, these null 237 results are not caused by the adjustment for multiple comparisons. Error bars denote \pm SEM. For 238 details see supplementary Table 1. 


\subsubsection{Pairwise correlations}

240 Next, pairwise correlations for all pairs of illusions and imagery scores were calculated for both

241 groups separately. Additionally, for the patients, SANS and SAPS scores were included into the 242 analysis. For the group of healthy controls, only three correlations were statistically significant 243 (Figure 3, upper panel; see supplementary table 2 for details): the Ponzo and Müller-Lyer, the 244 Ponzo "hallway" and Müller-Lyer, and the tilt and simultaneous contrast. For the group of 245 schizophrenia patients, the Ponzo and Müller-Lyer, the Ponzo "hallway" and Ebbinghaus, and 246 the White's and simultaneous contrast correlations were significant (Figure 3, lower panel; see 247 supplementary table 3 for details). Because we had a large number of comparisons ( 28 for the 248 controls and 45 for schizophrenia patients), we conducted a less conservative correction for 249 multiple comparisons than the Bonferroni correction, i.e., the Holm-Bonferroni correction. After 250 correction, only the correlation between the White's and the simultaneous contrast illusion 251 remained significant (Figure 3, three black stars; see supplementary table 8 for details relative to 252 the Holm-Bonferroni correction). On average, correlations including visual illusions and the 253 VVIQ score were slightly higher for the controls $(R=0.28 \pm 0.17)$ than for the patients $(R=$ $2540.20 \pm 0.19$ ), although that difference was not statistically significant (Table 1) 


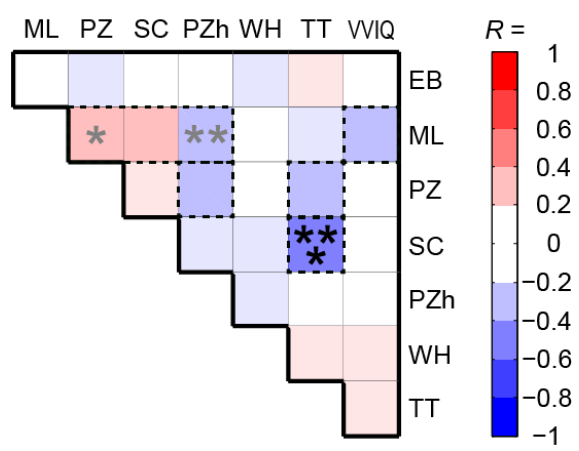

Schizophrenia patients (SZ)

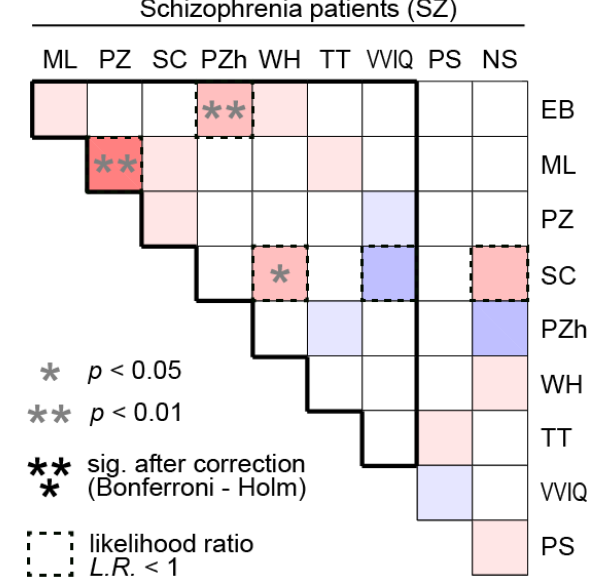

256 Figure 3. Correlograms for controls (HC, upper panel) and patients (SZ, lower panel). in Exp. 1 and

257 likelihood ratios for pairwise comparisons. Colors indicate Bravais-Pearson's $R$ correlation coefficient. Bold

258 lines delineate comparisons between the variables tested in both groups, i.e., the seven illusions and the 259 vividness of visual mental imagery score (VVIQ). Generally, correlations were low and only a few were 260 significant. Correlations were weaker amongst schizophrenia patients. Illusion magnitudes of the Ponzo 261 and Müller-Lyer illusions were significantly correlated in both groups. In addition in the control group, the 262 Ponzo "hallway" and the Müller-Lyer illusion, and the tilt and the simultaneous contrast illusions were 263 significantly correlated. In the schizophrenia group, the Ponzo "hallway" and the Ebbinghaus illusions, 264 and the simultaneous contrast and White's illusions were significantly correlated. The VVIQ score did not 265 correlate significantly with any other variable in none of the two groups. Similarly, SAPS and SANS scores 266 did not correlate significantly with any other variable amongst schizophrenia patients. Only the 267 correlations between the tilt and the simultaneous contrast illusion remained significant after correction for 268 multiple comparisons (Bonferroni-Holm; shown as three black stars). For details, see supplementary Tables 2692 and 3. A Bayesian analysis was used to evaluate the likelihood of existence or absence of relationship 
between pairwise comparisons. Bayesian likelihood ratios (L.R.) greater than one indicate support for the null hypothesis (supporting the absence of an effect) and values lower than one indicate support for the alternative hypothesis (supporting the existence of an effect; dashed line boxes).

\subsubsection{Bayes analysis}

274 We adopted a Bayesian approach in order to make statements beyond the usual "reject or fail to 275 reject the null hypothesis" outlined by Gallistel (2009) and implemented a method that was previously reported (Cappe et al., 2014). We measured for which comparisons the null hypothesis was more likely than the alternative hypothesis, given the data. The Bayesian analysis showed that the alternative hypothesis is more probable than the null hypothesis for all the pairwise comparisons that were shown to correlate significantly within the control group (Figure 3, dashed line; Ponzo - Müller-Lyer, Ponzo "hallway" - Müller-Lyer and tilt - simultaneous contrast) and amongst the schizophrenia patients (Ponzo - Müller-Lyer, Ponzo "hallway" - Ebbinghaus, Whites's - simultaneous contrast) and for some other pairwise comparisons in each group (controls, simultaneous contrast - Müller-Lyer, Ponzo "hallway" - Ponzo, tilt - Ponzo, VVIQMüller-Lyer; patients, VVIQ - simultaneous contrast, SANS - simultaneous contrast).

\subsubsection{Rank analysis}

One could expect that a participant highly susceptible to one illusion is also highly susceptible to other illusions. Similarly, if a given participant has a very vivid mental imagery, one could expect that the participant is strongly susceptible to all illusions. To the contrary, if there is no relationship between variables (here, illusion magnitudes and the VVIQ score), then participants' mean ranks are expected to be no different from chance. To test this hypothesis, we calculated each participant's rank for each variable. Then, we computed their mean ranks and compared the ranks with the ranks that would be expected from participants with random ranks (with random ranks averaged over 10,000 simulations). Results showed that neither the ranks of schizophrenia

294 patients $\left(\chi_{(18)}^{2}=0.36, p=1\right)$ nor of the controls $\left(\chi_{(18)}^{2}=0.48, p=1\right)$ were significantly different 295 from chance (Figure 4). 

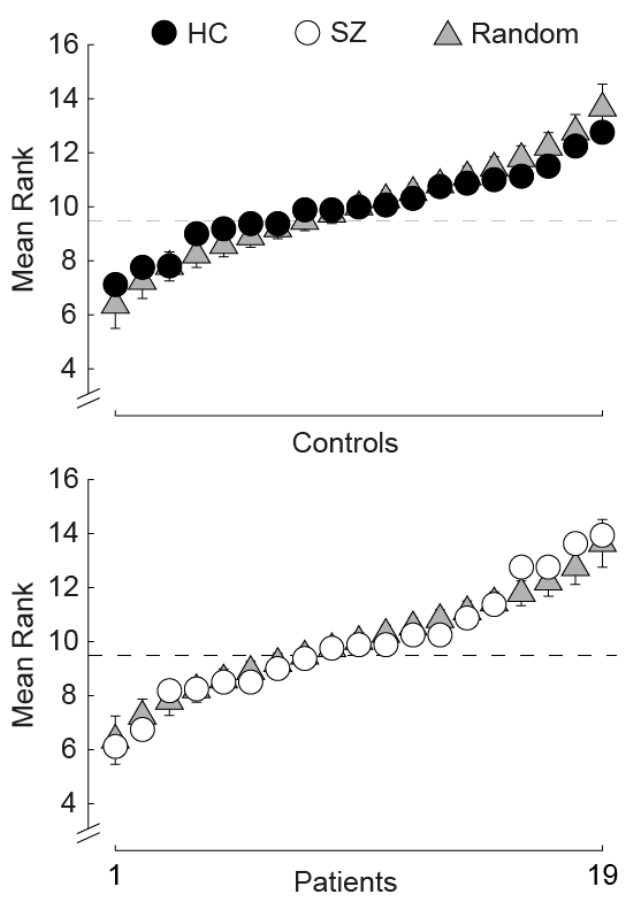

297 Figure 4. Ranks for each participant averaged over seven illusion magnitudes and the VVIQ score for the controls (upper panel, black disks) and the schizophrenia patients (lower panel, white disks) sorted by mean rank. Random simulated ranks, sorted by mean rank ( \pm 1 SD over 10,000 simulations, gray triangles). For both groups, i.e., patients and controls, mean ranks were not different from chance.

301

\section{Experiment 2}

302 Here, additionally to assessing the problem of the sample size in Exp. 1, we also asked whether different components of the same visual illusion are related and to which extent different illusions of the same kind are related. Thus, we measured the over- and the under-estimated components of the Ebbinghaus illusion separately (Figure 5; EBs: Ebbinghaus "small" and EBb: Ebbinghaus "big", respectively) and simultaneously (Figure 5; Ebbinghaus) and the susceptibility to three different variants of the Ponzo illusion (Ponzo, PZ; Ponzo "wide", PZw; and Ponzo "grid", PZg; Figure 5). Finally, for the schizophrenia patients, we included the medication type and its quantity to the analysis in order to test for their potential effects. 


\subsubsection{Stimuli}

312 We determined illusion magnitudes for ten visual illusions (Figure 5). Six of them, namely the

313 Ebbinghaus, the Müller-Lyer, the Ponzo, the White's, the simultaneous contrast and, the tilt

314 illusions were the same as in Exp. 1. Stimuli layout and proportions were the same as in Exp. 1

315 but scaled by a factor of $\approx 1.5$ because they were presented on a larger screen. Additionally, we

316 measured the Ebbinghaus illusion with small (EBs) and big (EBb) inducers separately, and two

317 different variants of the Ponzo illusion (PZw and PZg).

\subsubsection{Ebbinghaus Illusions (EB, EBs and EBb)}

319 Illusion susceptibility to three variants of the Ebbinghaus illusion was tested. First variant (EB) was the same as in Exp. 1. The second variant of the illusion (EBs) did not contain large inducers, thus the small inducers were surrounding the reference disk and the rightward, adjustable disk was not surrounded by any inducers. In the third variant $(\mathrm{EBb})$, large inducers were placed around the leftward reference disk while the adjustable disk was not surrounded by any inducers.

\subsubsection{Additional Ponzo illusions (PZw and PZg)}

In the Ponzo "wide" illusion (PZw), participants adjusted the upper horizontal line to match its length to the lower horizontal reference line. The reference was a 4.5 degrees long line. All lines were gray $\left(\approx 30.6 \mathrm{~cd} / \mathrm{m}^{2}\right)$. The initial length of the adjustable line was randomized from trial to trial and varied from 0 to 12 degrees. Both, the reference and the adjustable lines were centered on the vertical midline of the screen and were placed at 7.2 degrees from the screen's horizontal midline. The ends of the white diagonal lines (illusion inducers) were placed at 7.2 degrees from the screen's horizontal midline. The two upper and lower line ends of inducer lines were 6 and 18 degrees apart, respectively. In the Ponzo "grid" illusion (PZg), the reference stimulus was 5 degrees long, horizontal, lower line embed in a trapezoid which was embed in a grid aiming to 
induce perspective (Figure 5). The adjustable line was the horizontal, upper line embed in another trapezoid of the same size placed on the horizon line. Both trapezoids were isosceles trapezoids whose big (lower) and small (upper) edges were 15 and 9.2 degrees long, respectively.

The starting length of the adjustable line was randomized at each trial within a range of 0 to 22 degrees. Both, the reference and the adjustable lines were centered on the screen's vertical midline and were placed at horizontal distances from the screen's midline of 10 and 4.5 degrees, respectively. All lines had approximately the same luminance of $30.6 \mathrm{~cd} / \mathrm{m}^{2}$.
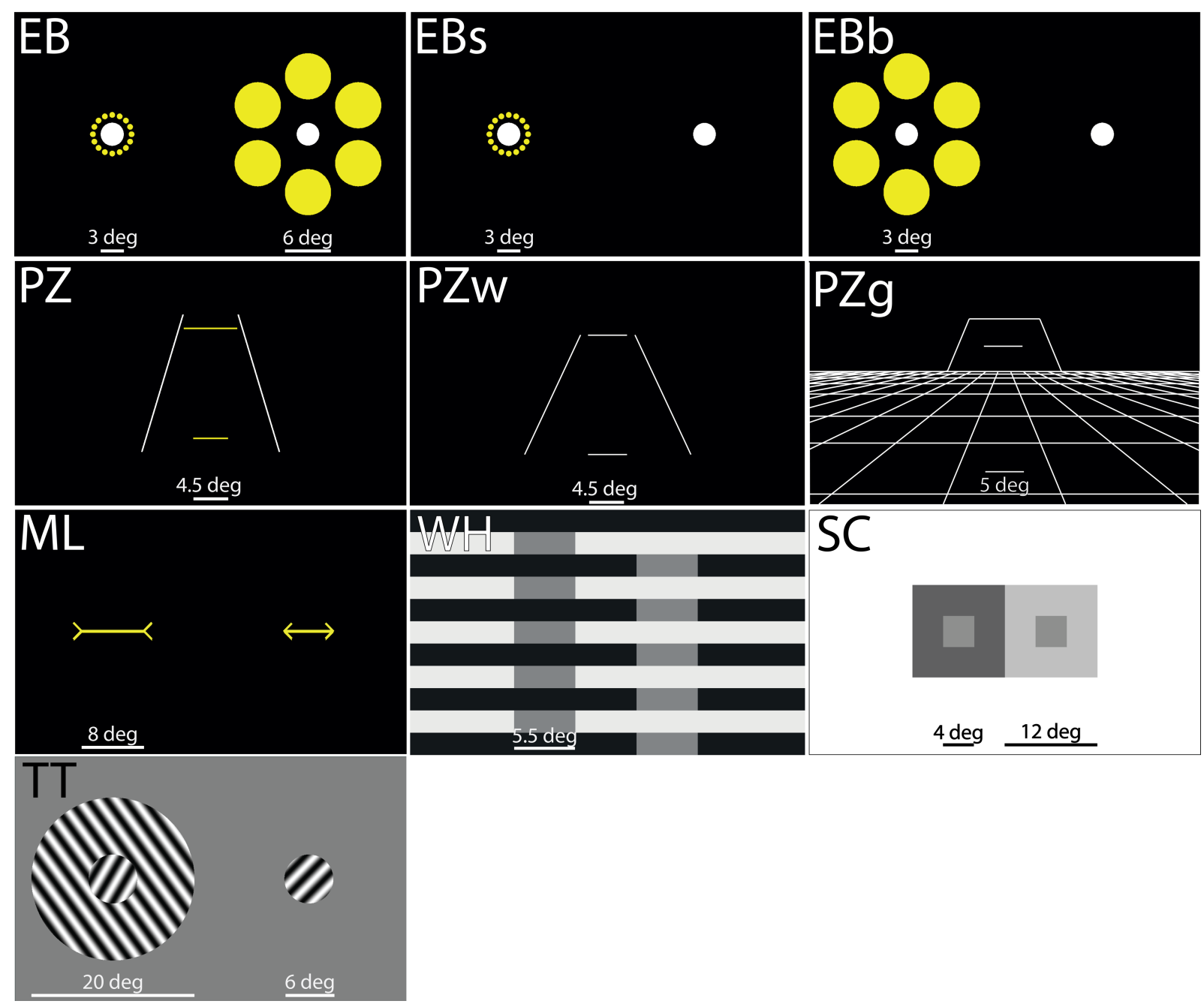

4 deg 12 deg

Figure 5. The susceptibility to ten visual illusions was tested in Exp. 2. We tested the susceptibility to the Ebbinghaus illusion with small and big inducers at the same time (EB) and separately (EBs and EBb). The susceptibility to three variants of the Ponzo illusions was measured: the same as in Exp. 1 (PZ), a wider version aiming to maximize the illusion $(\mathrm{PZw})$ and a version with an inducing perspective grid $(\mathrm{PZg})$. The 17 
347 Müller-Lyer (ML), White's (WH), simultaneous contrast (SC) and the tilt (TT) illusions were the same as

348 in Exp. 1. Likewise in Exp. 1, the task was to adjust the adjustable element of each illusion to its reference

349 by using the computer mouse.

\section{$350 \quad 3.1 .2$ Medication}

351 The medication type (MED) and its quantity (CPZ) were included in the part of the analysis.

352 Schizophrenia patients were classified depending on the medication type they receive as no 353 medication (0) typical (1), atypical (2), mixture of both (3), containing benzodiazepines (4).

\subsection{Results}

\subsubsection{Test-retest reliability}

356

Similarly to Exp. 1, the test-retest reliability was measured for each illusion by calculating

Bravais-Pearson's correlations between both trials for each illusion. All correlations were highly significant for both groups (Table 3). Except from the patient's White's illusion, all correlations remained significant after Bonferroni correction $(p=0.0025)$.

Table 3. Test-retest reliability in Exp. 2. Bravais-Pearson's $R$ correlations coefficients between the first and the second trial for ten visual illusions for schizophrenia patients (SZ, first row) and age-matched, healthy controls (HS, second row). All correlations were highly significant suggesting high reliability.

\begin{tabular}{lllllllllll}
\hline & EB & EBs & EBb & ML & PZ & PZw & PZg & SC & WH & TT \\
\hline SZ & $.55 \star \star \star$ & $.49 \star \star \star$ & $.58 \star \star \star$ & $.60 \star \star \star$ & $.61 \star \star \star$ & $.59 \star \star \star$ & $.86 \star \star \star$ & $.41 \star \star \star$ & $.35 \star \star$ & $.59 \star \star \star$ \\
HC & $.56 \star \star \star$ & $.72 \star \star \star$ & $.48 \star \star \star$ & $.45 \star \star \star$ & $.76 \star \star \star$ & $.61 \star \star \star$ & $.83 \star \star \star$ & $.85 \star \star \star$ & $.52 \star \star \star$ & $.48 \star \star \star$ \\
\hline & & & & & & & & & & \\
\hline
\end{tabular}

\subsubsection{Illusion magnitudes}

364 Individual illusion magnitudes were calculated by averaging the adjusted bias (or error) from both trials. We compared illusion magnitudes of patients and controls for each illusion by calculating independent samples $t$-tests without the assumption of equal variances. Satterthwaite's approximation for the effective degrees of freedom was calculated. Amongst ten comparisons, 
only one comparison was significantly different (Figure 6; see supplementary Table 4 for details).

369 Schizophrenia patients were less susceptible to the simultaneous contrast illusion (SC) than 370 controls $\left(t_{[103]}=3.33, p=0.0012\right)$. According to Cohen $(1988)$, that effect size is medium size $(d=$ 371 0.46). The effect remains significant after correcting for multiple comparisons $(\alpha=0.05 / 10=$ 3720.005 ; Bonferroni correction).
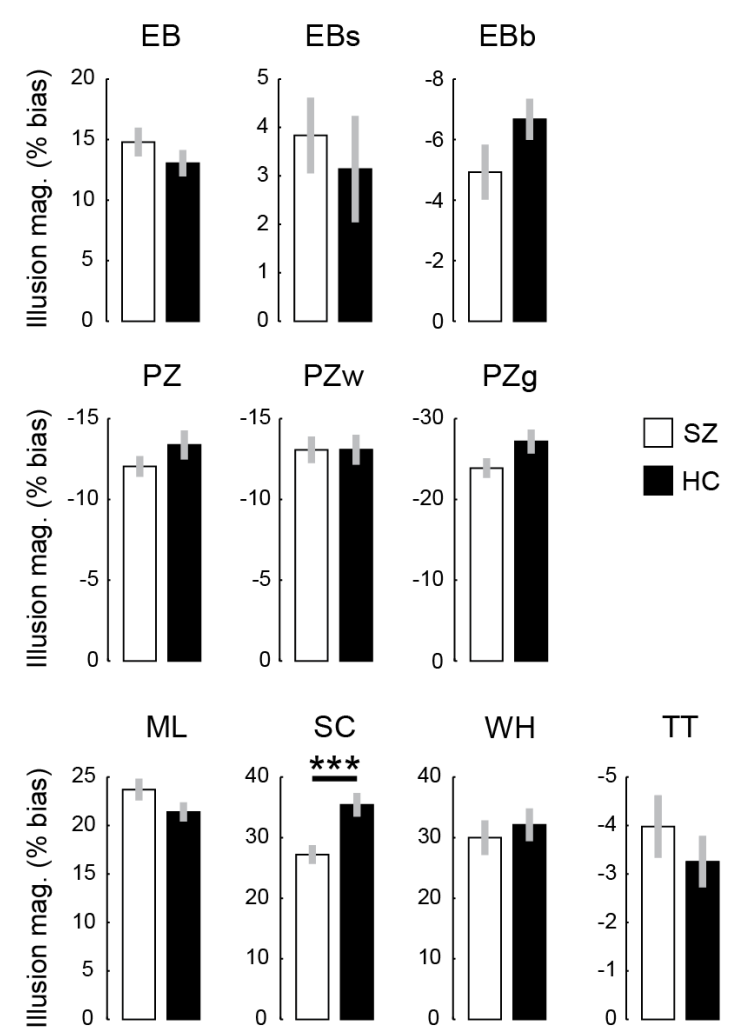

374 Figure 6. Exp 2: Mean illusion magnitudes as the percentage of bias (or error) for schizophrenia patients 375 (SZ, white) and healthy controls (HC, black). Note that scales for different illusions vary. The higher the absolute value of the magnitude, the higher the illusion susceptibility. Significant difference between patients and controls was found only for the simultaneous contrast illusion (SC). Patients were significantly less susceptible to the illusion than the controls $(p=0.0012, d=0.46)$ even after Bonferroni correction for multiple comparisons. For more details see supplementary Table 4. Error bars represent \pm SEM.

\subsubsection{Pairwise correlations}

381 For both groups, correlations were calculated in the same manner as in Exp. 1. For the controls, 382 correlations were calculated for the ten illusions and the age of the participants. For 
schizophrenia patients, the ten illusions, age, SANS and SAPS scores, the medication type

384 (MED) and its overall quantity, expressed as chlorpromazine-equivalent dosage (CPZ) were inter-correlated. Similarly to Exp.1, a large number of correlations was calculated (45 for the controls and 105 for schizophrenia patients), thus, we conducted a less conservative, HolmBonferroni correction for multiple comparisons instead of the Bonferroni correction. Expectedly, the three variants of the Ponzo illusion (PZ, PZw and PZg) were strongly and positively correlated for both groups (for details, see supplementary Tables 5 and 6). For the control group, the Ebbinghaus with small inducers (EBs) was strongly correlated to the Ebbinghaus containing both, the small and the big inducers (EB), the Müller-Lyer illusion (ML) and, the Ponzo illusion with the perspective grid $(\mathrm{PZg})$ but not to the Ebbinghaus with large inducers only $(\mathrm{EBb})$. All other comparisons were not significantly correlated after Holm-Bonferroni correction (see the supplementary table 8 for details concerning the corrected p-values).
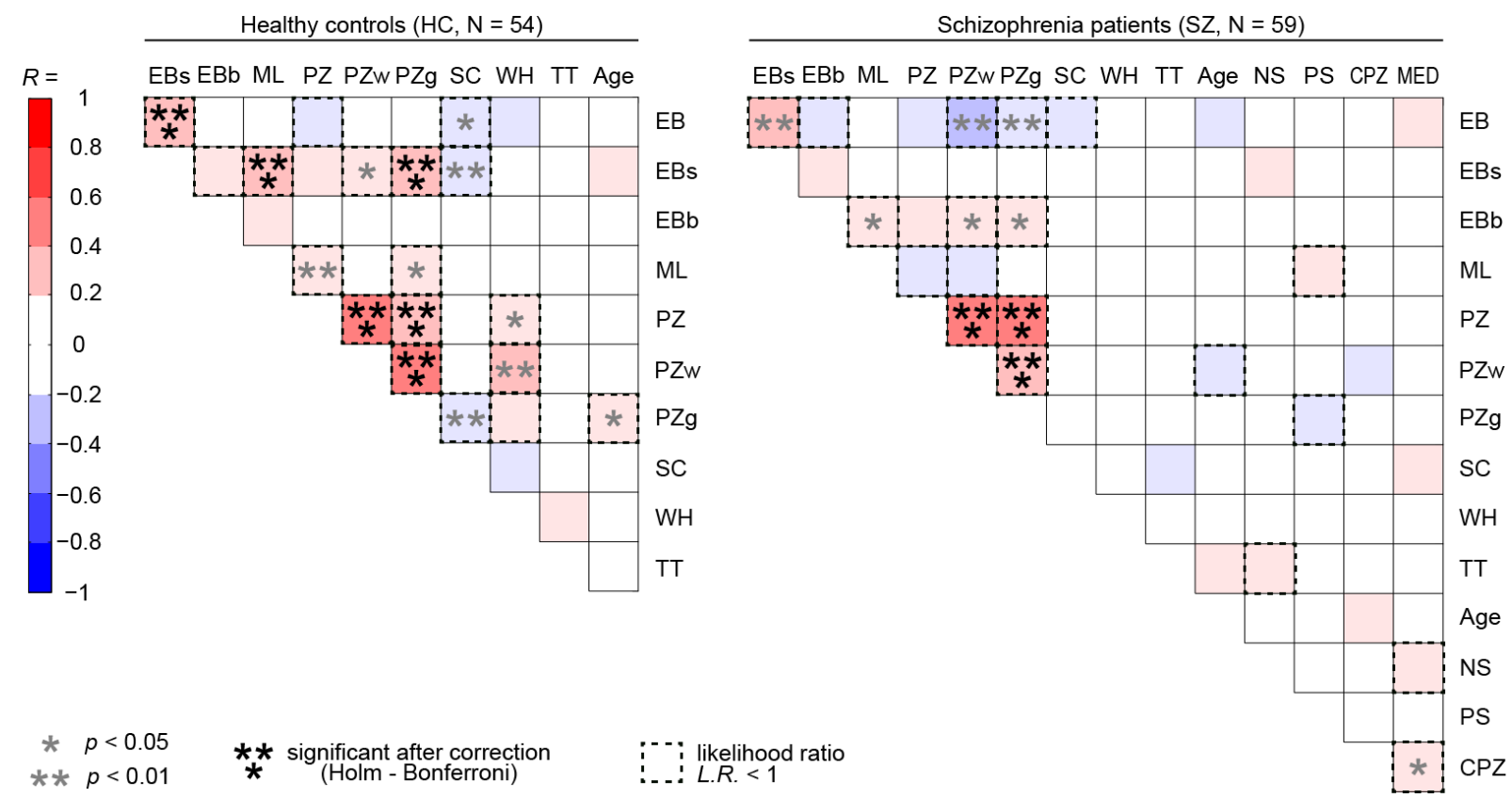

396 Figure 7. Correlograms for controls (left panel) and patients (right panel) in Exp. 2 and likelihood ratios for

397 pairwise comparisons. Colors indicate Bravais-Pearson's $R$ correlation coefficients. Significant correlations 398 are marked by gray stars, those of them remaining significant after correction for multiple comparisons 399 (Holm-Bonferroni) are marked by black stars. Unsurprisingly, all three Ponzo illusions (PZ, PZw and PZg) 
were strongly correlated in both groups. For the controls, the Ebbinghaus with small inducers (EBs) was strongly correlated to the Ebbinghaus with small and big inducers (EB), the Müller-Lyer illusion (ML), and, the Ponzo illusion with a perspective grid (PZg). All other correlations were not statistically significant after correction for multiple comparisons (Holm-Bonferroni). Amongst these significant correlations, only three were strong, namely the White's (WH) and Ponzo "wide" (PZw) correlation amongst the controls, and the Ebbinghaus (EB) and Ebbinghaus "small" (EBs), and the Ebbinghaus (EB) and the Ponzo "wide" (PZw) correlations amongst the patients.. Similarly to Exp. 1, negative (NS) and positive (PS) symptoms scores assessed with SANS and SAPS inventories, respectively, did not correlate significantly with any other variable. Interestingly, the type of medication (MED), or its overall amount (CPZ) were not related to any other variable. For all the details, see supplementary Tables 5 and 6 . Bayesian likelihood ratios (L.R.) greater than one (dashed line boxes) indicate support for the null hypothesis (supporting the absence

411 of an effect) and values lower than one indicate support for the alternative hypothesis (supporting the existence of an effect). For most of the comparisons, the L.R.s support the absence of effects.

\subsubsection{Bayesian analysis}

414 We adopted the same Bayesian analysis on all pairwise comparisons as in Exp.1 (Cappe et al., 2014; Gallistel, 2009). For both patients and controls, we measured for which comparisons the null hypothesis was more likely than the alternative hypothesis, given the data. The alternative

417 hypothesis, (suggesting existence of an effect) was more probable than the null hypothesis 418 (suggesting the absence of an effect) for all significantly correlated pairs within both groups 419 (Figure 7, dashed line boxes). Additionally, Bayes analysis suggested the existence of an effect for three other, non-correlated comparisons amongst controls and nine amongst patients.

\subsubsection{Rank analysis}

As in Exp. 1, we calculated mean ranks for controls and patients in order to verify if some participants are generally more or less susceptible to visual illusions. Results showed that neither the ranks of schizophrenia patients $\left(\chi_{(18)}^{2}=0.36, p=1\right)$ nor of the controls $\left(\chi_{(18)}^{2}=0.48, p=1\right)$ were significantly different from simulated, random ranks averaged over 10,000 simulations

426 (Figure 8). 

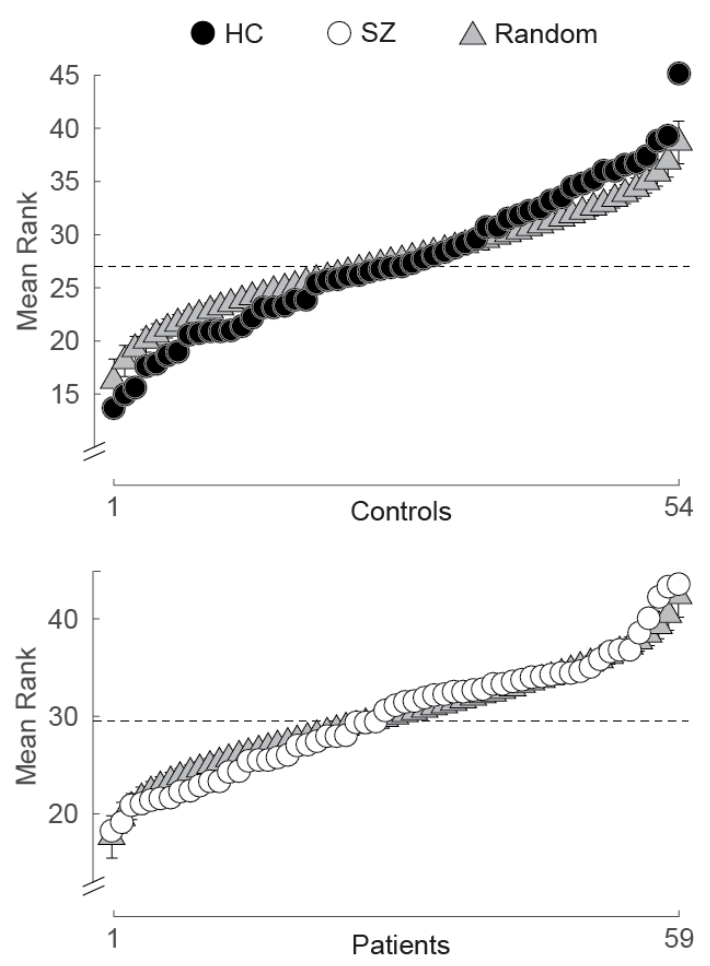

428 Figure 8. Mean ranks for each control participant (upper panel, black disks) and patient (lower panel, white disks) averaged over ten illusion magnitudes and sorted by mean rank. Random simulated ranks, sorted by mean rank ( \pm 1 SD over 10,000 simulations, gray triangles). Mean ranks for patients and controls were not different from chance.

\subsubsection{Principal component analysis (PCA)}

433 In order to reduce the dimensionality of our data and to identify potential hidden factors, we 434 conducted a principal component analysis (PCA). PCA included both patients and controls and 435 was conducted on eleven variables, i.e., the ten illusions magnitudes and age. Two principal 436 components (PC1 and PC2) were identified by the means of the scree plot inspection (Figure 9a). 437 The PC1, explaining $23.8 \%$ of the variability in the data was mainly composed by the three 438 Ponzo illusions (PZg, PZ and PZw) with respective loadings of 0.52, 0.51 and 0.51 (Figure 9c, 439 left panel; Figure 9d). The PC2, explained $17 \%$ of the variance and was dominated by loadings of $440 \mathrm{~EB}, \mathrm{EBs}$ and ML illusion, with loadings of $0.56,0.51$ and 0.40 , respectively (Figure 9c, right 441 panel; Figure 9d). For more details see supplementary Table 7. Importantly, patients did not 442 differ from controls in their eigenvalues for PC1 and PC2 (Figure 9b), suggesting that the 
cumulated explained variance $(\mathrm{PC} 1+\mathrm{PC} 2=40.8 \%)$ was unrelated to the belonging of the

444 participants to the patient or control group. Age did not load importantly on any of the two 445 principal components.

a

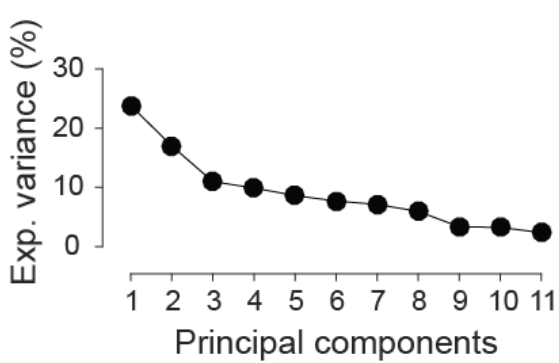

b

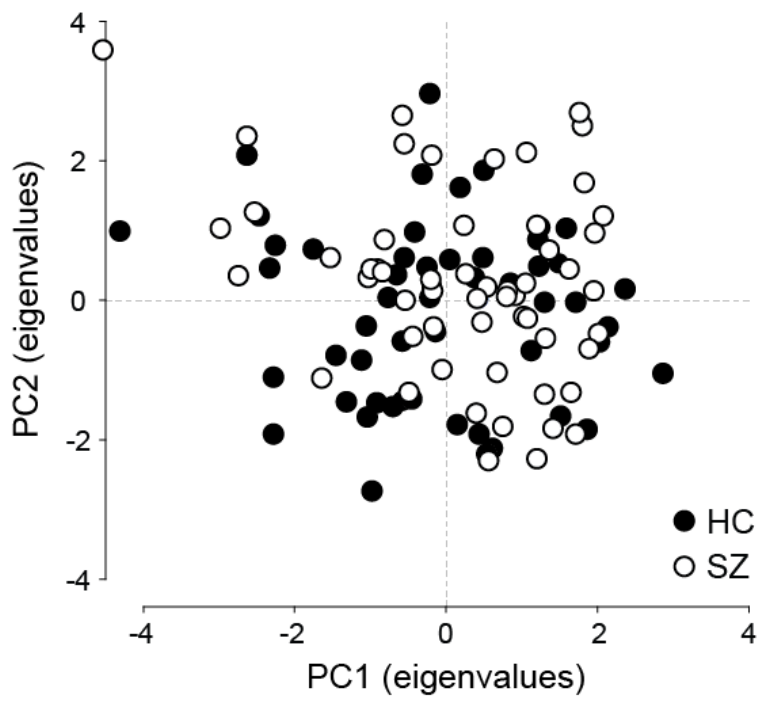

Loadings PC1

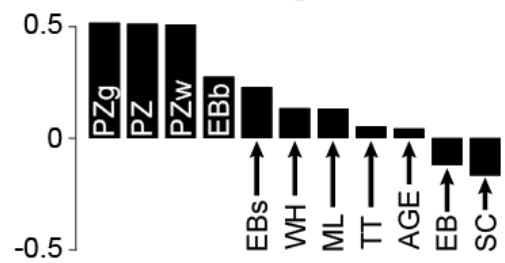

Loadings PC2

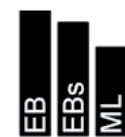

$\uparrow \uparrow_{\omega} \uparrow \uparrow \uparrow$

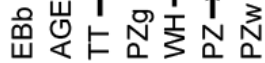

446

447

448

449

450

451

452

453

454

455

Figure 9. A principal component analysis (PCA) was performed on the data of patients (SZ) and controls (HC). The ten illusion magnitudes and the age of each observer were included in the PCA. (a) Two principal components were identified (PC1 and $\mathrm{PC} 2)$ on the basis of scree plot inspection, accounting for $23.8 \%$ and $17 \%$ of the variability of the data. (b) Eigenvalue score plot for all the observers for PC1 and PC2. Neither PC1 nor PC2 was able to separate patients (white disks) from the controls (black disks), suggesting that most of the variability in the data (cumulated explained variance for PC1 and PC2, i.e., 40.8\%) was unrelated to the disease. These results suggest that other factors than schizophrenia account for that data variability. (c) Expectedly, component coefficients (or loadings) for PC1 were mainly composed by the three Ponzo illusions (PZg, PZ and PZw). The PC2, was mainly composed by the Ebbinghaus with 
small inducers (EBs), Ebbinghaus with both, small and big inducers (EB), and the Müller-Lyer illusion

457 (ML). (d) Loading plot for the two principal components. Surprisingly, the Ebbinghaus illusion with big 458 inducers (EBb) was more related to the Ponzo illusions rather than other two Ebbinghaus illusions (EB and 459 EBs) whereas the Müller-Lyer illusion was related to the Ebbinghaus with small (EBs) and with both (EB) 460 inducers but not to the Ponzo illusions. For more details see supplementary Table 7.

\section{Discussion}

462 Since the early days of schizophrenia research, it has been reported that patients perceive the world in a different phenomenological way than healthy controls (Bleuler, 1950; Butler et al., 2008; Sergi et al., 2006). Here, we tested whether patients perceive illusions differently than controls.

Illusion magnitude and the quest for a common factor. We tested 19 and 59 patients and 19 and 54 controls in Exp. 1 and 2, respectively. First, we found that illusion magnitudes were roughly the same in patients and controls. Second, we found very few significant correlations between the illusions in both groups and experiments. In the first experiment, we found only one significant correlation between the tilt and the simultaneous contrast illusion after we corrected for multiple comparisons (Holm-Bonferroni). In the second experiment with a higher power, the three Ponzo illusions correlated significantly for both the patients and the controls. In addition, the Ebbinghaus illusion with small inducers (EBs) was correlated to the Ponzo "grid" (PZg), to the Müller-Lyer, and unsurprisingly to the Ebbinghaus illusion with both, the big and small inducers (EB). However, other spatial illusions, such as the Ponzo and the Ebbinghaus with small and big inducers (EB) illusion did not significantly correlate with each other, in line with previous findings (Schwarzkopf et al., 2011). In general, except for these significant correlations, only 14 out of the remaining 81 correlations were significant without correction for multiple comparisons

479 (6 for patients, 8 for controls; none of the significant correlations were the same for patients and controls; Figure 7). Thus, correlations between different visual illusions are sparse and this is even 
more true for schizophrenia patients, which is in line with previous results (Tibber et al., 2013;

482 Yang et al., 2013). For instance, Tibber and colleagues (2013) found only 1 significant correlation 483 out of 8 comparisons. Yang et al., (2013) did not find any significant inter-illusion correlations for 484 four measures. In summary, illusion magnitudes do not strongly differ between patients and 485 controls. In addition, there are not more correlations in the patients than in the controls. Hence, the disease does not seem to induce a common factor for illusion perception. The perception of illusions seems to be roughly intact in the patients.

Numerous theories have proposed that the perception of illusions should be different in patients and controls. For example, it was proposed that schizophrenia patients have different visual priors than controls, making their vision more veridical and leading to a decrease in illusion magnitude (Fletcher and Frith, 2009). Similarly, it has also been argued that patients have a "failure to attenuate sensory precision", which means they cannot call upon their prior experiences to interpret the current stimuli (Frith and Friston, 2013). Other theories have suggested a deficit in contextual modulation and surround inhibition in patients, which might be the consequence of a weaker interaction between adjacent neurons and, therefore, a weaker gain control in schizophrenia (e.g., Butler et al., 2008; Phillips and Silverstein, 2013; Tadin et al., 2006; Tibber et al., 2013; Yang et al., 2013). Potential mechanisms for these deficits might include reduced modulation of cortical responses in the primary visual cortex (Seymour et al., 2013) or a reduction in the population of receptive fields in the early visual cortex (Anderson et al., 2017). As a consequence, patients tend to be less affected by helpful or deleterious contexts (Dakin et al., 2005; Robol et al., 2013) and for this reason, illusion magnitudes might be smaller. Our results do not support these claims, since we found the perception of illusions is largely intact in the patients, both in terms of illusions magnitudes and their correlation structure.

Mental imagery, positive symptoms, and illusions strength. In addition, we found no correlations between illusion magnitudes and positive or negative symptoms, as determined by the SAPS and SANS, respectively, despite a wide range of symptoms in our patients (Table 1). We also found 
only weak correlations between mental imagery and illusion magnitudes. VVIQ scores were actually higher in controls than patients but the effect was not significant (Table 1). The VVIQ scores of the control group in this study were slightly higher than the scores of the healthy participants in a previous study (Grzeczkowski et al., 2017; 118 \pm 25 vs. $113 \pm 28$ ). Taken together,

511 it seems that illusion magnitudes and vividness of mental imagery are comparable to the results of healthy controls.

Test-retest reliability and statistical power. Our null results cannot be explained by poor test-retest reliability or low statistical power. First, our test-retest correlations were significant for most of the illusions in both experiments (Tables 2 and 3). For the Ponzo illusion in Exp. 1 for example, our test-retest reliability was $R=0.89$ for the patients and $R=0.98$ for the controls. In Exp. 2, all ten illusions showed significant test-retest correlations for both groups. Moreover, we found significant correlations between illusions that were expected to correlate, such as the Ponzo illusions in Exp 2 (Figure 7). Therefore, our method seems to be sensitive to observe differences when differences exist.

521 Second, with 59 patients, we had $99 \%, 65 \%$, and $12 \%$ power to detect large $(R=0.5)$, medium $(R$ $522=0.3)$, and small $(R=0.1)$ effect sizes, respectively (Cohen,1988). Third, our null results are 523 supported by a Bayes analysis showing that the acceptation of the null hypothesis is more likely 524 than its rejection for most pairwise comparisons (Figure 3 and Figure 7, boxes with dashed line). 525 A rank analysis further confirmed that there are no participants who are more or less susceptible to visual illusions in general (Figure 4 and Figure 8).

527 We like to mention that even higher test-retests might potentially be achieved by using 2 AFC tasks and more trials. For example, test-retest reliability of our healthy controls was smaller than the one of healthy controls in a previous study by Schwarzkopf et al., (2011), which used a binary procedure and had more trials per illusion. Nevertheless, Ponzo and Ebbinghaus illusions did not 
531 correlate in that study either. Here, we refrained from using a binary method to reduce

532 attentional demands and to keep the experiment short.

533 Why do results differ in the literature? As mentioned, various studies have found increased or 534 decreased illusion magnitudes in the patients as compared to controls, while other studies have 535 found non-significant results (for a review, see King et al., 2016, Notredame et al., 2014). We 536 found a higher variance in the performance of the patients compared to the one of controls. This 537 may be one reason why previous results are mixed (for a review, see King et al., 2016; 538 Notredame et al., 2014). Another reason for mixed results may be the response measure used. In 539 our study, we used a mouse adjustment procedure, which allows participants to demonstrate 540 quickly and directly how they perceive the illusion. In most other studies, staircase procedures 541 were employed. Potentially, this procedure requires attentional and decisional resources that 542 might be deficient in the patients (King et al., 2016; Chkonia et al., 2010). Finally, as mentioned 543 above, samples are small in most studies and samples in schizophrenia research are usually 544 heterogeneous because of the heterogeneity of the disease, differences in medication and 545 hospitalization, and genetic differences of the different populations.

546 Limitations. We used an adjustment method that allowed us to rapidly and directly probe the 547 susceptibility to illusions within a few trials. We used two trials per illusion and for most of the 548 illusions, the correlations between these two trials were strong and significant (Table 2 and Table 549 3). It remains an open question whether better estimates of illusions strength could be achieved by 550 the method of constant stimuli, which may increase both inter-illusion correlations and test-retest 551 reliability. In addition, it may be worth to increase the number of adjustments per illusion to 552 obtain better estimates. We measured the susceptibility to only seven but frequently used 553 illusions. It remains an open question whether also for other illusions low correlations are found.

554 Conclusions. Illusion magnitudes of patients were similar to the ones of controls. In addition, we 555 found only weak correlations between illusions magnitudes in both patients and controls. We 
think that it is important to publish such null results and not only significant results, as it is common practice (Francis, 2012a, 2012b; Francis et al., 2014). Otherwise, the impression may occur that patients are deteriorated in most paradigms, which is not the case. We have previously reported that contextual modulation (Roinishvili et al., 2015) and complex motion perception (Lauffs et al., 2016) are intact in schizophrenia patients, and here we report that patients perceive visual illusions in a similar way to controls.

\section{Acknowledgements}

We would like to thank Marc Repnow for technical support; Aaron Clarke, Aline Cretenoud,

Victoire Gorden and Pornbhussorn Kanchanakanok for help in setting up the experiment; Aline

Cretenoud for feedback on the manuscript. This work was funded by the project "Learning from

Delayed and Sparse Feedback" (CRSII2_147636), the National Centre of Competence in

Research (NCCR) SYNAPSY grant (565557 NCCR SYN P14 LPSY), and the Swiss National

Science Foundation grant (320030_135741).

\section{References}

570

Anderson, E.J., Tibber, M.S., Schwarzkopf, D.S., Shergill, S.S., Fernandez-Egea, E., Rees, G., Dakin, S.C., 2017. Visual Population Receptive Fields in People with Schizophrenia Have Reduced Inhibitory Surrounds. J. Neurosci. 37, 1546-1556. doi:10.1523/JNEUROSCI.3620-15.2016

Andreasen N.C. (1984a). Scale for the Assessment of Negative Symptoms (SANS), University of Iowa, Iowa City

Andreasen N.C. (1984b). Scale for the Assessment of Positive Symptoms (SAPS), University of Iowa, Iowa City

Bach, M., 1996. The Freiburg Visual Acuity test--automatic measurement of visual acuity. Optom. Vis. Sci. 73, 49-53.

Barch, D.M., Carter, C.S., Dakin, S.C., Gold, J., Luck, S.J., MacDonald, A., Ragland, J.D., 

Silverstein, S., Strauss, M.E., 2012. The Clinical Translation of a Measure of Gain Control: The Contrast-Contrast Effect Task. Schizophr. Bull. 38, 135-143. doi:10.1093/schbul/sbr154

Benson, T.L., Park, S., 2013. Exceptional visuospatial imagery in schizophrenia; implications for madness and creativity. Front. Hum. Neurosci. 7, 756. doi:10.3389/fnhum.2013.00756

Bleuler, E., 1950. Dementia praecox or the group of schizophrenias. International Universities Press, Oxford.

Bosten, J.M., Mollon, J.D., 2010. Is there a general trait of susceptibility to simultaneous contrast? Vision Res. 50, 1656-1664. doi:10.1016/J.VISRES.2010.05.012

Bracha, H.S., Wolkowitz, O.M., Lohr, J.B., Karson, C.N., Bigelow, L.B., 1989. High prevalence of visual hallucinations in research subjects with chronic schizophrenia. Am. J. Psychiatry 146, 526-8. doi:10.1176/ajp.146.4.526

Brainard, D.H., 1997. The Psychophysics Toolbox. Spat. Vis. 10, 433-436.

Brébion, G., Amador, X., David, A., Malaspina, D., Sharif, Z., Gorman, J.M., 2000. Positive symptomatology and source-monitoring failure in schizophrenia - An analysis of symptomspecific effects. Psychiatry Res. 95, 119-131. doi:10.1016/S0165-1781(00)00174-8

Brébion, G., Smith, M.J., Gorman, J.M., Amador, X., 1997. Discrimination accuracy and decision biases in different types of reality monitoring in schizophrenia. J. Nerv. Ment. Dis. $185,247-253$.

Brune, M., 2005. Theory of Mind; in Schizophrenia: A Review of the Literature. Schizophr. Bull. 31, 21-42. doi:10.1093/schbul/sbi002

Burton, L.J., Fogarty, G.J., 2003. The factor structure of visual imagery and spatial abilities. Intelligence 31, 289-318. doi:10.1016/S0160-2896(02)00139-3

Butler, P.D., Silverstein, S.M., Dakin, S.C., 2008. Visual perception and its impairment in schizophrenia. Biol. Psychiatry 64, 40-7. doi:10.1016/j.biopsych.2008.03.023

Capozzoli, N.J., Marsh, D., 1994. Schizophrenia and geometric illusions. Report of perceptual distortion. Schizophr. Res. 13, 87-9.

Cappe, C., Clarke, A., Mohr, C., Herzog, M., 2014. Is there a common factor for vision? J. Vis. 14, 1-11. doi:10.1167/14.8.4.doi 
610

611

612

613

614

615

616

617

618

619

620

621

622

623

624

625

626

627

628

629

630

631

632

633

634

635

636

637

638

639

Chen, Y., McBain, R., Norton, D., Ongur, D., 2011. Schizophrenia patients show augmented spatial frame illusion for visual and visuomotor tasks. Neuroscience 172, 419-426. doi:10.1016/j.neuroscience.2010.10.039

Chkonia, E., Roinishvili, M., Herzog, M.H., Brand, A., 2010. First-order relatives of schizophrenic patients are not impaired in the Continuous Performance Test. J. Clin. Exp. Neuropsychol. 32, 481-486. doi:10.1080/13803390903201777

Cohen, J., 1992. Quantitative Methods in Psychology. Psychol. Bull. 112, 155-159.

Cohen, J., 1988. Statistical power analysis for the behavioral sciences. Stat. Power Anal. Behav. Sci. doi:10.1234/12345678

Crawford, T.J., Hamm, J.P., Kean, M., Schmechtig, A., Kumari, V., Anilkumar, A.P., Ettinger, U., 2010. The perception of real and illusory motion in schizophrenia. Neuropsychologia 48, 3121-3127. doi:10.1016/J.NEUROPSYCHOLOGIA.2010.06.027

Dakin, S., Carlin, P., Hemsley, D., 2005. Weak suppression of visual context in chronic schizophrenia [2]. Curr. Biol. 15, 822-824. doi:10.1016/j.cub.2005.10.015

Dima, D., Roiser, J.P., Dietrich, D.E., Bonnemann, C., Lanfermann, H., Emrich, H.M., Dillo, W., 2009. Understanding why patients with schizophrenia do not perceive the hollow-mask illusion using dynamic causal modelling. Neuroimage 46, 1180-1186. doi:10.1016/j.neuroimage.2009.03.033

Diržius, E., Liutkevičius, J., Zukauskaité, G., Leskauskas, D., Bulatov, A., 2, Aleksandr BuLATOv, 2013. Müller-Lyer illusion manifestation peculiarities among people with schizophrenia and bipolar disorders: a pilot study. Biol. Psychiatry Psychopharmacol. 15, $43-46$.

Emrich, H.M., Leweke, F.M., Schneider, U., 1997. Towards a Cannabinoid Hypothesis of Schizophrenia: Cognitive Impairments Due to Dysregulation of the Endogenous Cannabinoid System. Pharmacol. Biochem. Behav. 56, 803-807. doi:10.1016/S00913057(96)00426-1

Fletcher, P.C., Frith, C.D., 2009. Perceiving is believing: a Bayesian approach to explaining the positive symptoms of schizophrenia. Nat. Rev. Neurosci. 10, 48-58. doi:10.1038/nrn2536

Ford, J.M., Palzes, V.A., Roach, B.J., Potkin, S.G., Van Erp, T.G.M., Turner, J.A., Mueller, 
McEwen, S.C., Mathalon, D.H., 2015. Visual hallucinations are associated with hyperconnectivity between the amygdala and visual cortex in people with a diagnosis of schizophrenia. Schizophr. Bull. 41, 223-232. doi:10.1093/schbul/sbu031

Francis, G., 2012a. Publication bias and the failure of replication in experimental psychology. Psychon. Bull. Rev. 19, 975-991. doi:DOI 10.3758/s13423-012-0322

Francis, G., 2012b. The Psychology of Replication and Replication in Psychology. Perspect. Psychol. Sci. 7, 585-594. doi:10.1177/1745691612459520

Francis, G., Tanzman, J., Matthews, W.J., 2014. Excess success for psychology articles in the journal Science. PLoS One 9, 1-15. doi:10.1371/journal.pone.0114255

Frith, C.D., Corcoran, R., 1996. Exploring 'theory of mind' in people with schizophrenia. Psychol. Med. 26, 521. doi:10.1017/S0033291700035601

Frith, C.D., Friston, K.J., 2013. False perceptions and false beliefs: Understanding schizophrenia. Neurosci. Hum. Pers. New Perspect. Hum. Act. Scripta Varia 121, 1-15.

Gallistel, C.R., 2009. The Importance of Proving the Null. Psychol. Rev. 116, 439-453. doi:10.1037/a0015251.The

Goghari, V.M., Harrow, M., 2016. Twenty year multi-follow-up of different types of hallucinations in schizophrenia, schizoaffective disorder, bipolar disorder, and depression. Schizophr. Res. doi:10.1016/j.schres.2016.06.027

Grzeczkowski, L., Clarke, A.M., Francis, G., Mast, F.W., Herzog, M.H., 2017. About individual differences in vision. Vision Res. 141, 282-292. doi:10.1016/J.VISRES.2016.10.006

Kantrowitz, J.T., Butler, P.D., Schecter, I., Silipo, G., Javitt, D.C., 2009. Seeing the world dimly: The impact of early visual deficits on visual experience in schizophrenia. Schizophr. Bull. 35, 1085-1094. doi:10.1093/schbul/sbp100

Keane B. P., Silverstein S. M., Wang Y., Papathomas T. V. (2013). Reduced depth inversion illusions in schizophrenia are state-specific and occur for multiple object types and viewing conditions. J. Abnorm. Psychol. 122, 506

King, D.J., Hodgekins, J., Chouinard, P.A., Chouinard, V.-A., Sperandio, I., 2016. A review of abnormalities in the perception of visual illusions in schizophrenia. Psychon. Bull. Rev. 1- 
670

671

672

673

674

675

676

677

678

679

680

681

682

683

684

685

686

687

688

689

690

691

692

693

694

695

696

697

698

Lauffs, M.M., Shaqiri, A., Brand, A., Roinishvili, M., Chkonia, E., Öğmen, H., Herzog, M.H., 2016. Local versus global and retinotopic versus non-retinotopic motion processing in schizophrenia patients. Psychiatry Res. 246, 461-465. doi:10.1016/j.psychres.2016.09.049

Letourneau, J.E., 1974. The Oppel-Kundt and the Müller-Lyer Illusions among Schizophrenics. Percept. Mot. Skills 39, 775-778. doi:10.2466/pms.1974.39.2.775

Marks, D.F., 1973. Visual imagery differences and eye movements in the recall of pictures. Percept. Psychophys. 14, 407-412. doi:10.3758/BF03211175

Notredame, C.-E., Pins, D., Deneve, S., Jardri, R., 2014. What visual illusions teach us about schizophrenia. Front. Integr. Neurosci. 8. doi:10.3389/fnint.2014.00063

Oertel, V., Rotarska-Jagiela, A., van de Ven, V., Haenschel, C., Grube, M., Stangier, U., Maurer, K., Linden, D.E.J., 2009. Mental imagery vividness as a trait marker across the schizophrenia spectrum. Psychiatry Res. 167, 1-11. doi:10.1016/j.psychres.2007.12.008

Parnas, J., Vianin, P., Saebye, D., Jansson, L., Volmer Larsen, A., Bovet, P., 2001. Visual binding abilities in the initial and advanced stages of schizophrenia. Acta Psychiatr. Scand. 103, 171-180. doi:10.1034/j.1600-0447.2001.00160.x

Pelli, D.G., 1997. The VideoToolbox software for visual psychophysics: transforming numbers into movies. Spat. Vis. 10, 437-442. doi:10.1163/156856897X00366

Perlstein, W.M., Carter, C.S., Barch, D.M., Baird, J.W., 1998. The Stroop task and attention deficits in schizophrenia: A critical evaluation of card and single-trial Stroop methodologies. Neuropsychology 12, 414-425. doi:10.1037/0894-4105.12.3.414

Phillips, W.A., Silverstein, S.M., 2013. The coherent organization of mental life depends on mechanisms for context-sensitive gain-control that are impaired in schizophrenia. Front. Psychol. 4, 307. doi:10.3389/fpsyg.2013.00307

Power, R.A., Steinberg, S., Bjornsdottir, G., Rietveld, C.A., Abdellaoui, A., Nivard, M.M., Johannesson, M., Galesloot, T.E., Hottenga, J.J., Willemsen, G., Cesarini, D., Benjamin, D.J., Magnusson, P.K.E., Ullén, F., Tiemeier, H., Hofman, A., van Rooij, F.J.A., Walters, G.B., Sigurdsson, E., Thorgeirsson, T.E., Ingason, A., Helgason, A., Kong, A., Kiemeney, L.A., Koellinger, P., Boomsma, D.I., Gudbjartsson, D., Stefansson, H., Stefansson, K., 2015. Polygenic risk scores for schizophrenia and bipolar disorder predict creativity. Nat. 
700

701

702

703

704

705

706

707

708

709

710

711

712

713

714

715

716

717

718

Robol, V., Tibber, M.S., Anderson, E.J., Bobin, T., Carlin, P., Shergill, S.S., Dakin, S.C., 2013. Reduced Crowding and Poor Contour Detection in Schizophrenia Are Consistent with Weak Surround Inhibition. PLoS One 8, e60951. doi:10.1371/journal.pone.0060951

Roinishvili, M., Cappe, C., Shaqiri, A., Brand, A., Rürup, L., Chkonia, E., Herzog, M.H., 2015. Crowding, grouping, and gain control in schizophrenia. Psychiatry Res. 226, 441-445. doi:10.1016/j.psychres.2015.01.009

Sack, A.T., van de Ven, V.G., Etschenberg, S., Schatz, D., Linden, D.E.J., 2005. Enhanced vividness of mental imagery as a trait marker of schizophrenia? Schizophr. Bull. 31, 97-104. doi:10.1093/schbul/sbi011

Sanders, L.L.O., de Millas, W., Heinz, A., Kathmann, N., Sterzer, P., 2013. Apparent motion perception in patients with paranoid schizophrenia. Eur. Arch. Psychiatry Clin. Neurosci. 263, 233-239. doi:10.1007/s00406-012-0344-5

Schneider, U., Borsutzky, M., Seifert, J., Leweke, F.M., Huber, T.J., Rollnik, J.D., Emrich, H.M., 2002. Reduced binocular depth inversion in schizophrenic patients. Schizophr. Res. 53, 101-8. doi:10.1016/S0920-9964(00)00172-9

Schwarzkopf, D.S., Song, C., Rees, G., 2011. The surface area of human V1 predicts the subjective experience of object size. Nat. Neurosci. 14, 28-30. doi:10.1038/nn.2706

Sergi, M.J., Rassovsky, Y., Nuechterlein, K.H., Green, M.F., 2006. Social perception as a mediator of the influence of early visual processing on functional status in schizophrenia. Am. J. Psychiatry 163, 448-54. doi:10.1176/appi.ajp.163.3.448

Seymour, K., Stein, T., Sanders, L.L.O., Guggenmos, M., Theophil, I., Sterzer, P., 2013. Altered Contextual Modulation of Primary Visual Cortex Responses in Schizophrenia. Neuropsychopharmacology 38, 2607-2612. doi:10.1038/npp.2013.168

Shaqiri, A., Clarke, A., Kunchulia, M., Herzig, D., Pilz, K., Herzog, M., 2015. The effects of aging on perception and cognition. J. Vis. 15, 802. doi:10.1167/15.12.802

Spencer, K.M., Ghorashi, S., 2014. Oscillatory dynamics of Gestalt perception in schizophrenia revisited. Front. Psychol. 5, 68. doi:10.3389/fpsyg.2014.00068

Tadin, D., Kim, J., Doop, M.L., Gibson, C., Lappin, J.S., Blake, R., Park, S., 2006. Weakened 

26, 11403-11412. doi:10.1523/jneurosci.2592-06.2006

730

Tam, W.C., Sewell, K.W., Deng, H.C., 1998. Information processing in schizophrenia and bipolar disorder: a discriminant analysis. J. Nerv. Ment. Dis. 186, 597-603.

Tibber, M.S., Anderson, E.J., Bobin, T., Antonova, E., Seabright, A., Wright, B., Carlin, P., Shergill, S.S., Dakin, S.C., 2013. Visual surround suppression in schizophrenia. Front. Psychol. 4, 1-13. doi:10.3389/fpsyg.2013.00088

Uhlhaas, P.J., Phillips, W.A., Mitchell, G., Silverstein, S.M., 2006. Perceptual grouping in disorganized schizophrenia. Psychiatry Res. 145, 105-117. doi:10.1016/j.psychres.2005.10.016

Waters, F., Collerton, D., ffytche, D.H., Jardri, R., Pins, D., Dudley, R., Blom, J.D., Mosimann, U.P., Eperjesi, F., Ford, S., Larøi, F., 2014. Visual Hallucinations in the Psychosis Spectrum and Comparative Information From Neurodegenerative Disorders and Eye Disease. Schizophr. Bull. 40, S233-S245. doi:10.1093/schbul/sbu036

Weckowicz, T.E., Witney, G., 1960. The Müller-Lyer Illusion in Schizophrenic Patients. J. Ment. Sci. 106, 1002-1007. doi:10.1192/bjp.106.444.1002

Yang, E., Tadin, D., Glasser, D.M., Hong, S.W., Blake, R., Park, S., 2013. Visual context processing in schizophrenia. Clin. Psychol. Sci. 1, 5-15. doi:10.1177/2167702612464618 